equation. But there are many ways that negative intraplewral pressure (i.e., interstitial pressure) can result in unbalanced Starling forces favouring pulmonary oedema formation.

The authors agree that hypoxaemia is a major contributing factor in most cases of negative pressure pulmonary oedema. However, hypoxaemia cannot explain the development of pulmonary oedema in a controlled experimental environment where animals are exposed to high-grade airway obstruction and hypoxaemia is prevented with high inspired oxygen concentrations. Therefore, we conclude that hypoxaemia cannot be the major factor and certainly is not the sole factor responsible for the development of negative pressure pulmonary oedema.

Scott A. Lang MD FRCPC

Saskatoon

\section{Laryngeal mask airway and visualisation of vocal cords during thyroid surgery}

To the Editor:

Laryngeal nerve damage is an established complication of thyroid surgery.' Tracheal intubation makes the intraoperative direct visualization of the vocal cords impossible and attempts to detect laryngeal nerve damage postoperatively are in vain as the damage has been done and may be permanent.

The use of laryngeal mask airway for thyroid surgery enables direct visualisation of the vocal cords intraoperatively using a fibreoptic paediatric bronchoscope. My practice is to induce anaesthesia IV with propofol 2.5 $\mathrm{mg} \cdot \mathrm{kg}^{-1}$, insert a laryngeal mask airway, and maintain anaesthesia with enflurane, nitrous oxide and oxygen with spontaneous ventilation.

Excessive traction or minor trauma of the recurrent laryngeal nerves will usually cause mild laryngospasm and stridor and warn of the impending damage. The laryngospasm usually settles spontaneously on release of the traction on the nerve or by increasing the depth of anaesthesia.

In patients with distorted anatomy of the neck structures it is often difficult or impossible to identify anatomical structures including laryngeal nerves. In such cases stimulation of the suspected laryngeal nerves with an electrical nerve stimulator and visualising the movement of the vocal cords using the above technique will aid identification of the laryngeal nerves and measures can then be adopted to avoid trauma to the nerves during dissection.

This method can be used in place of postoperative postextubation laryngoscopy. The vocal cords can easily be visualized at the end of the operation which avoids the need for direct laryngoscopy. ${ }^{2}$

T.M. Akhtar MB FFARCS(I)

Leeds, England

\section{REFERENCES}

1 Atkinson RS, Rushman GB, Alfred Lee J. A Synopsis of Anaesthesia. 10th cd London: Wright 1987; 455.

2 Sarma VJ. Cord examination after thyroidectomy. Anaesthesia, 1989; 44: 531. 